\title{
Performance analysis of fixed-gain AF dual-hop relaying systems over Nakagami- $m$ fading channels in the presence of interference
}

\author{
Fawaz S Al-Qahtani ${ }^{*}$, Caijun Zhong ${ }^{2}$, Khalid A Qaraqe ${ }^{1}$, Hussein Alnuweiri ${ }^{1}$ and Tharm Ratnarajah ${ }^{3}$
}

\begin{abstract}
This article presents an analytical investigation on the performance of interference-limited fixed-gain amplify-andforward dual-hop relaying systems over Nakagami-m fading channels. Assuming the fading parameter $m$ of the two hop channels being integer, we derive a closed-form expression of the cumulative distribution function of a new type of random variables involving a number of independent gamma random variables, based on which, the outage performance and symbol error rate of the system are examined, and two important performance metrics at the high signal-to-noise ratio regime, namely, diversity order and coding gain, are characterized. Moreover, expressions of the general moments of the end-to-end signal-to-interference-and-noise ratio are derived and then applied in the analysis of the ergodic capacity of the system. In addition, the impact of interference power distribution on the ergodic capacity of the system is studied with the aid of a majorization result. Our findings suggest that the diversity of the system is limited by the hop experiencing severer fading, and co-channel interferences do not reduce the diversity order of the system, instead, they degrade the outage performance by affecting the coding gain of the system.
\end{abstract}

Keywords: amplify-and-forward relaying, co-channel interference, cooperative communications, Nakagami-m fading, performance analysis

\section{Introduction}

Dual-hop relaying transmission, as a means to improve the throughput and extend the coverage of the wireless communication system, has recently received enormous interests in the context of cooperative communications $[1,2]$, where an intermediate mobile device acts as a relay node and helps forward the signal received from the source node to the intended destination node. Among various relaying protocols proposed in [2], amplify-and-forward (AF) relaying scheme, where the relay node simply forwards a scaled version of the received signal, has received a great deal of attention because of its simplicity and ease of implementation.

Depending on the availability of instantaneous channel state information (CSI) at the relay node, AF relaying scheme generally falls into two categories, i.e.

\footnotetext{
* Correspondence: fawaz.al-qahtani@qatar.tamu.edu

'Electrical \& Computer Engineering Program, Texas A\&M University at Qatar, Doha, Qatar

Full list of author information is available at the end of the article
}

variable-gain relaying [3] and fixed-gain AF relaying [4]. A large number of studies has been conducted to understand the performance of AF dual-hop systems in various popular fading channel models [5-12]. In $[5,6]$, the outage probability and error rate of dual-hop AF systems were studied in Rayleigh fading channels, while [7-10] investigated the performance of dual-hop AF systems in Nakagami- $m$ fading channels. The performance of dual-hop AF system in more general fading channels was considered in $[11,12]$. While these studies have greatly improved our knowledge on the topic, they all assume that the communication takes place in an interference-free environment. However, because of aggressive reuse of frequency, wireless communications are generally affected by co-channel interference (CCI) $[13,14]$. Hence, there is a strong need to understand the impact of $\mathrm{CCI}$ on the performance of dual-hop systems.

In the presence of CCI, there has been very few studies on the performance of dual-hop systems, most in 
Rayleigh fading channels. In $[15,16]$, the outage probability of opportunistic decode-and-forward relaying dual-hop system was studied, and in [17], the outage probability of a fixed-gain AF relaying system with interference-limited destination has been investigated. The study [18] analyzed the outage and error performance of dual-hop AF relaying with interference at the relay node, while $[19,20]$ studied the more general model where both the relay and the destination are corrupted by CCIs. In [21], the authors investigated fixed-gain AF relaying system in the presence of CCIs at the relay and destination assuming Rayleigh faded dual-hop channels with Rician fading interfering channels.

While Rayleigh fading channel is an important channel model, understanding the performance of dual-hop systems in the more general Nakagami- $m$ fading channels has also received much attention [7-10]. Assuming Nakagami- $m$ fading channels, [22] investigated the outage and average symbol error rate (SER) of variable-gain AF dualhop systems with interference-limited relay and noisy destination. While [22] improved our knowledge on the topic, the impact of CCIs at the destination and the effect of fixed-gain relaying scheme in Nakagami- $m$ fading channels have not been well understood. Motivated by this, in this article, we present a detailed analytical investigation on the performance of fixed-gain AF dual-hop relaying system with noisy relay and interference-limited destination in Nakagami- $m$ fading channels.

The main contribution of the article is the derivation of the cumulative distribution function (c.d.f.) of a new type of random variable involving sum of multiple-independent gamma random variables. Based on which, we present closed-form expressions for the outage probability and average SER of the system. A closed-form expression for the general moments of the end-to-end signal-to-interference-and-noise ratio (SINR) is derived, which is then applied to investigate the ergodic capacity of the system. Moreover, to gain further valuable insights into the system, we also provide simple expressions for outage probability of the system at high signalto-noise ratio (SNR) regime, which enable efficient characterization of the diversity order and coding gain achieved by the system.

The remainder of this article is organized as follows: Section 2 introduces the system model. Section 3 presents closed-form analytical expressions for various important system performance metrics, i.e., outage probability, SER and ergodic capacity. In Section 4, numerical results are provided to verify the accuracy of our analysis and examine the impact of CCI on the performance of dual-hop AF relaying systems. Finally, we conclude the article in Section 5.

\section{System Model}

Consider a dual-hop relay where a source node S transmits to a destination node $\mathrm{D}$ with the assistance of a relay node $R$. The entire communication takes place in two separate phases. In the first phase, $\mathrm{S}$ transmits the signal to $R$ and hence the received signal at the relay node can be written as

$$
y_{\mathrm{r}}=h_{\mathrm{sr}} x_{0}+n_{\mathrm{sr}}
$$

where $x_{0}$ is the transmitted symbol with $\mathbb{E}\left\{\left|x_{0}\right|^{2}\right\}=P_{0}$ and $h_{s r}$ is the channel coefficient or the S-R link, $n_{\text {sr }} \sim \mathcal{C N}\left(0, N_{1}\right)$ denotes the additive white Gaussian noise, and $\mathbb{E}\{\cdot\}$ denotes the expectation operation. In the second phase, the received signal at $\mathrm{R}$ is first scaled with a fixed gain $G \triangleq \sqrt{\frac{P_{r}}{P_{0} \Omega_{1}+N_{1}}}$ and then forwarded to $\mathrm{D}$. The signal at the destination is corrupted by interfering signals from $N$ co-channel interferers $\left\{x_{i}\right\}_{i=1}^{N}$, each with an average power of $P_{i}$. As in [17], we consider the interference-limited destination case, therefore, the signal received at the destination can be expressed as

$$
y_{d}=G h_{\mathrm{rd}} h_{\mathrm{sr}} x_{0}+G h_{r d} n_{\mathrm{sr}}+\sum_{i=1}^{N} h_{i} x_{i}
$$

where $h_{\mathrm{rd}}$ denotes the channel coefficient for the R-D link, $\left\{h_{i}\right\}_{i}^{N}$ are the channel coefficients from interferers to D. We assume that the channel gains $\left|h_{\mathrm{sr}}\right|^{2}$ and $\left|h_{\mathrm{rd}}\right|^{2}$ follow the gamma distribution with different fading parameters $1 / \Omega_{1}, 1 / \Omega_{2}$ and fading severity parameters $m_{1}, m_{2}$, respectively. Similarly, the channel gains $\left|h_{i}\right|^{2}, i$ $=1, \ldots, N$, are assumed to follow independent gamma distribution with parameters $m_{I_{i}}$ and $1 / \Omega_{I_{i}}$.

After some algebraic manipulations, the end-to-end SINR can be expressed as

$$
\gamma_{\mathrm{d}}=\frac{G^{2} P_{0}\left|h_{\mathrm{rd}}\right|^{2}\left|h_{\mathrm{sr}}\right|^{2}}{G^{2}\left|h_{\mathrm{rd}}\right|^{2} N_{1}+\sum_{i=1}^{N} P_{i}\left|h_{i}\right|^{2}}=\left(\frac{P_{0}}{N_{1}}\right)\left(\frac{X_{1} X_{2}}{X_{2}+\frac{1}{G^{2} N 1} Y}\right),
$$

where $X_{1} \triangleq\left|h_{\mathrm{sr}}\right|^{2}, X_{2} \triangleq\left|h_{\mathrm{rd}}\right|^{2}$ and $Y \triangleq \sum_{i=1}^{N}\left|g_{i}\right|^{2}$, where $\left|g_{i}\right|^{2}=P_{i}\left|h_{i}\right|^{2}$ is gamma distributed with parameters $m_{I_{i}}$ and $1 / \bar{\gamma}_{I_{i}}$, with $\bar{\gamma}_{I_{i}}=P_{i} \Omega_{I_{i}}$.

\section{End-to-end performance}

In this section, we present a detailed performance investigation of interference-limited dual-hop AF relaying system in Nakagami- $m$ fading channels by studying several important performance measures, i.e., outage probability, general moments of the end-to-end SINR, 
average SER and ergodic capacity of the system. For notational conveniencewe define $\rho_{1}=\frac{P_{0}}{N_{1}}$, and $\rho_{2}=\frac{P_{r}}{N_{1}}$.

Before getting into the details, we find it is convenient to first present the following theorem.

Theorem 1: Let $X=\frac{U_{1} U_{2}}{U_{2}+u Y}$, where $U_{i}, i=1,2$, are gamma distributed random variables with parameters $m_{1}, 1 / \Omega_{1}$ and $m_{2}, 1 / \Omega_{2}$, respectively. $Y=\sum_{i=1}^{N} Y_{i}$ with $Y_{i}, i=1, \ldots, N$ being independent gamma random variables with parameter $m_{I_{i}}$ and $1 / \bar{\gamma}_{I_{i}} . u$ is a positive constant. Then the c.d.f. of $X$ is given by ${ }^{a}$

$$
\begin{array}{r}
\mathcal{F}_{X}(x)=1-\left(\frac{m_{2} \bar{\gamma}_{l_{k}}}{\Omega_{2} m_{l_{k}}}\right)^{m_{2}} \exp \left(-\frac{m_{1} x}{\Omega_{1}}\right)^{m_{1}-1} \sum_{i=0} \frac{1}{i !}\left(\frac{m_{1} x}{\Omega_{1}}\right)^{i} \sum_{j=0}^{i}\left(\begin{array}{l}
i \\
j
\end{array}\right) u^{j} \\
\sum_{k=1}^{N} \sum_{t=1}^{m_{l_{k}}} \frac{\beta_{k}^{t-1} \Gamma\left(a_{1}\right) \Gamma\left(a_{2}\right)}{(t-1) ! \Gamma\left(m_{2}\right)}\left(\frac{u m_{1} x}{\Omega_{1}}\right)^{m_{2}-j} U\left(a_{1}, a_{3}, \frac{u m_{1} m_{2} x \bar{\gamma}_{I_{k}}}{\Omega_{1} \Omega_{2} m_{l_{k}}}\right),
\end{array}
$$

where

$$
a_{1}=m_{I_{k}}+m_{2}, a_{2}=m_{I_{k}}+j,
$$

$a_{3}=m_{2}-j+1, m_{I_{k}}, \bar{\gamma}_{I_{k}}$ and $\beta_{t}^{k}$ are defined in Appendix $\mathrm{A}$, and $U(a, b, x)$ is the confluent hypergeometric function of the second kind [[23], Eq. 9.210.2].

Proof: See Appendix A.

\subsection{Outage probability}

The outage probability is an important system performance metric, and is defined as the probability that the instantaneous SINR $\gamma_{\mathrm{d}}$ falls below a predefined threshold, $\gamma_{\text {th }}{ }^{\text {b }}$ Mathematically, the outage probability of the end-to-end SINR $\gamma_{\mathrm{d}}$ can be presented as

$$
P_{\text {out }}\left(\gamma_{\text {th }}\right)=\operatorname{Pr}\left(\gamma_{\mathrm{d}}<\gamma_{\text {th }}\right)=\operatorname{Pr}\left(\frac{X_{1} X_{2}}{X_{2}+\frac{1}{G^{2} N 1} Y}<\frac{\gamma_{\text {th }}}{\rho_{1}}\right)(5)
$$

To this end, utilizing Theorem 1 , the outage probability of the end to end SINR $\gamma_{\mathrm{d}}$ can be obtained as

$$
\begin{aligned}
& P_{\text {out }}\left(\gamma_{\text {th }}\right)=1-\left(\frac{m_{2} \bar{\gamma}_{I_{k}}}{\Omega_{2} m_{l_{k}}}\right)^{m_{2}} \exp \left(-\frac{m_{1} \gamma_{\text {th }}}{\Omega_{1} \rho_{1}}\right)^{m_{1}-1} \sum_{i=0}^{1} \frac{1}{i !}\left(\frac{m_{1} \gamma_{\text {th }}}{\Omega_{1} \rho_{1}}\right)^{i} \sum_{j=0}^{i}\left(\begin{array}{l}
i \\
j
\end{array}\right)\left(\frac{\rho_{1} \Omega_{1}+1}{\rho_{2} N_{1}}\right)^{j} \\
& \sum_{k=1}^{N} \sum_{t=1}^{m_{l_{k}}} \frac{\beta_{k}^{t-1} \Gamma\left(a_{1}\right) \Gamma\left(a_{2}\right)}{(t-1) ! \Gamma\left(m_{2}\right)}\left(\frac{m_{1} \gamma_{\mathrm{th}}\left(\rho_{1} \Omega_{1}+1\right)}{\Omega_{1} \rho_{1} \rho_{2} N_{1}}\right)^{m_{2}-j} U\left(a_{1}, a_{3}, \frac{m_{1} m_{2} \gamma_{\mathrm{th}}\left(\rho_{1} \Omega_{1}+1\right) \bar{\gamma}_{I_{k}}}{\Omega_{1} \Omega_{2} m_{I_{k}} \rho_{1} \rho_{2} N_{1}}\right)
\end{aligned}
$$

For the special case, where the interfering channels experience the same level of fading and the average received interfering powers at the destination are the same, we have the following simple result.

Corollary 1: For the case where $m_{I_{i}}=m_{I}$ and $\bar{\gamma}_{I_{i}}=\bar{\gamma}_{I}, i=1, \ldots, N$, the outage probability expression reduces to

$$
\begin{aligned}
& P_{\text {out }}\left(\gamma_{\mathrm{th}}\right)=1-\left(\frac{m_{2} \bar{\gamma}_{I}}{\Omega_{2} m_{I}}\right)^{m_{2}} \exp \left(-\frac{m_{1} \gamma_{\mathrm{th}}}{\Omega_{1} \rho_{1}}\right) \sum_{i=0}^{m_{1}-1} \frac{1}{i !}\left(\frac{m_{1} \gamma_{\mathrm{th}}}{\Omega_{1} \rho_{1}}\right)^{i} \sum_{k=0}^{i}\left(\begin{array}{l}
i \\
k
\end{array}\right)\left(\frac{\rho_{1} \Omega_{1}+1}{\rho_{2} N_{1}}\right)^{k} \\
& \frac{\Gamma\left(b_{1}\right) \Gamma\left(b_{2}\right)}{\Gamma\left(N m_{I}\right) \Gamma\left(m_{2}\right)}\left(\frac{m_{1} \gamma_{\mathrm{th}}\left(\rho_{1} \Omega_{1}+1\right)}{\Omega_{1} \rho_{1} \rho_{2} N_{1}}\right)^{m_{2}-k} U\left(b_{1}, a_{3}, \frac{m_{1} m_{2} \gamma_{\mathrm{th}}\left(\rho_{1} \Omega_{1}+1\right) \bar{\gamma}_{I}}{\Omega_{1} \Omega_{2} m_{I} \rho_{1} \rho_{2} N_{1}}\right)
\end{aligned}
$$

where $b_{1}=N m_{I}+m_{2}$, and $b_{2}=N m_{I}+j$.

Proof: The proof is straightforward, and thus omitted.
Note that, for the case $m_{1}=m_{2}=m_{I}=1$, i.e., Rayleigh fading channels, (7) reduces to the results presented in [17].

While the above expression offers an efficient way to evaluate the outage probability of the system, the expression itself is in general too complex to give any insight. Therefore, it is of great interest to look into the high SNR regime, where simple expressions can be obtained.

Theorem 2: At the high SNR regime, the outage probability of the system can be approximated as

(1) If $\rho_{1} \rightarrow \infty$,

$$
P_{\text {out }}\left(\gamma_{\mathrm{th}}\right) \approx 1-\left(\frac{m_{1} m_{2} \gamma_{\mathrm{th}} \bar{\gamma}_{l}}{\rho_{2} \Omega_{2} N_{1} m_{l}}\right)^{m_{2}} \sum_{i=0}^{m_{1}-1} \frac{1}{i !} \frac{\Gamma\left(b_{1}\right) \Gamma\left(N m_{l}+i\right)}{\Gamma\left(N m_{l}\right) \Gamma\left(m_{2}\right)} U\left(b_{1}, m_{2}-i+1, \frac{m_{1} m_{2} \gamma_{\mathrm{th}} \bar{\gamma}_{l}}{\Omega_{2} m_{1} \rho_{2} N_{1}}\right)
$$

(2) If $\rho_{1} \rightarrow \infty$,

$$
P_{\text {out }}\left(\gamma_{\mathrm{th}}\right) \approx 1-\exp \left(-\frac{m_{1} \gamma_{\mathrm{th}}}{\Omega_{1} \rho_{1}}\right) \sum_{i=0}^{m_{1}-1} \frac{1}{i !}\left(\frac{m_{1} \gamma_{\mathrm{th}}}{\Omega_{1} \rho_{1}}\right)^{i} .
$$

(3) If $\rho_{2}=\mu \rho_{1}$ and $\rho_{1} \rightarrow \infty$,

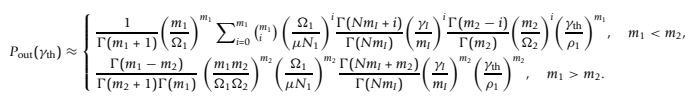

Proof: See Appendix B.

Theorem 2 gives some intuitive results regarding the outage probability of the interference-limited dual-hop AF relaying system. For instance, the outage performance of the system is affected by both hops, increasing the power at either the transmit or relay node while keeping the other power fixed, the outage probability reaches an error floor. In particular, if the available power at the relay node is sufficiently large, then the outage performance is completely determined mined by the performance of the first hop transmission as shown in Equation 9. In addition, when both the transmit and relay powers become large, the diversity order achieved by the system is limited by the weaker links of the first and second hop channels. Moreover, the CCIs do not reduce the diversity order of the system, instead, it degrades the outage performance of the system by affecting the coding gain of the system.

\subsection{General moments of the end-to-end SINR}

The general moments are important performance measures which can be used to obtain the end-to-end average SINR, variance and amount of fading (AoF). By definition, the $n$th moment of $\gamma_{d}$ is given by $\mathcal{I}(n)=n \int_{0}^{\infty} \gamma^{n-1}\left(1-\mathcal{F}_{\gamma_{\mathrm{d}}}(\gamma)\right) d \gamma$. With the help of [[23], Eq. 7.621.6] and the c.d.f. expression given in Equation 6, we can express the $n$th moments of the end-to-end SIR $\gamma_{\mathrm{d}}$ as 


$$
\begin{aligned}
\mathcal{I}(n) & =\left(\frac{m_{2} \bar{\gamma}_{k}}{\Omega_{2} m_{l_{k}}}\right)^{m_{2}} \sum_{i=0}^{m_{1}-1} \frac{n}{i !}\left(\frac{m_{1}}{\Omega_{1} \rho_{1}}\right)^{i} \sum_{j=0}^{i}\left(\begin{array}{l}
i \\
j
\end{array}\right)\left(\frac{\rho_{1} \Omega_{1}+1}{\rho_{2} N_{1}}\right)^{j} \sum_{k=1}^{N} \sum_{t=1}^{m_{l_{k}}} \frac{\beta_{k}^{t-1} \Gamma\left(a_{1}\right)}{(t-1) ! \Gamma\left(m_{2}\right)} \\
& \times\left(\frac{m_{1}\left(\rho_{1} \Omega_{1}+1\right)}{\Omega_{1} \rho_{1} \rho_{2} N_{1}}\right)^{m_{2}-j}\left(\frac{\Omega_{1} \Omega_{2} m_{I_{k}} \rho_{1} \rho_{2} N_{1}}{m_{1} m_{2}\left(\rho_{1} \Omega_{1}+1\right) \bar{\gamma}_{k}}\right)^{\beta_{1}} \Upsilon_{1}
\end{aligned}
$$

where $\Upsilon_{1}$ is given by

$$
\Upsilon_{1}=\left\{\begin{array}{l}
\frac{\Gamma\left(\beta_{1}\right) \Gamma(n+i)}{\Gamma\left(a_{1}+n+i\right)}{ }_{2} F_{1}\left(\beta, n+i ; a_{1}+n+i ; 1-A\right), \quad|1-\mathrm{A}|<1, \\
\frac{\Gamma\left(\beta_{1}\right)(n+n) i}{\Gamma\left(a_{1}+n+i\right)}\left(\frac{m_{2}\left(\rho_{1} \Omega_{1}+1\right) \bar{\gamma}_{l_{k}}}{\Omega_{2} m_{l_{k}} \rho_{2} N_{1}}\right)_{2}{ }_{2} F_{1}\left(a_{1}, \beta_{1} ; a_{1}+n+i ; 1-\frac{1}{A}\right), \mathrm{A}>1 / 2,
\end{array}\right.
$$

with $A=\frac{\Omega_{2} m_{I_{k}} \rho_{2} N_{1}}{m_{2}\left(\rho_{1} \Omega_{1}+1\right) \bar{\gamma}_{I_{k}}}$ and $\beta_{1}=n+m_{2}+i+j$.

For the special case, where $m_{I_{k}}=m_{I}$ and $\bar{\gamma}_{I_{k}}=\bar{\gamma}_{I}$, for $k=1, \ldots, N$, the $n$th moments of the end-to-end SINR $\gamma_{\mathrm{d}}$ admits a simpler expression

$$
\begin{aligned}
\mathcal{I}(n) & =\left(\frac{m_{2} \bar{\gamma}_{1}}{\Omega_{2} m_{l}}\right)^{m_{2}} \sum_{i=0}^{m_{1}-1} \frac{1}{i !}\left(\frac{m_{1}}{\Omega_{1} \rho_{1}}\right)^{i} \sum_{j=0}^{i}\left(\begin{array}{l}
i \\
j
\end{array}\right)\left(\frac{\rho_{1} \Omega_{1}+1}{\rho_{2} N_{1}}\right)^{j} \frac{n \Gamma\left(b_{1}\right) \Gamma\left(b_{2}\right)}{\Gamma\left(N m_{l}\right) \Gamma\left(m_{2}\right)}\left(\frac{m_{1}\left(\rho_{1} \Omega_{1}+1\right)}{\Omega_{1} \rho_{1} \rho_{2} N_{1}}\right)^{m_{2}-} \\
& \times\left(\frac{\Omega_{1} \Omega_{2} m_{1} \rho_{1} \rho_{2} N_{1}}{m_{1} m_{2}\left(\rho_{1} \Omega_{1}+1\right) \bar{\gamma}_{I}}\right)^{\beta_{1}} \Upsilon_{2},
\end{aligned}
$$

where $\Upsilon_{2}$ is given by

$$
\Upsilon_{2}=\left\{\begin{array}{l}
\frac{\Gamma(\beta) \Gamma(n+i)}{\Gamma\left(b_{1}+n+i\right) 2} F_{1}\left(\beta, n+i ; b_{1}+n+i ; 1-A\right),|1-\mathrm{A}|<1, \\
\frac{\Gamma\left(\beta_{1}\right) \Gamma(n+i)}{\Gamma\left(b_{1}+n+i\right)}\left(\frac{\left.m_{2}\left(\rho_{1} \Omega_{1}+1\right)\right)_{1}}{\Omega_{2} m_{1} \rho_{2} N_{1}}\right)^{\beta_{1}}{ }_{2} F_{1}\left(b_{1}, \beta_{1} ; b_{1}+n+i ; 1-\frac{1}{A}\right), \mathrm{A}>1 / 2 .
\end{array}\right.
$$

As a direct application, the average end-to-end SINR can be obtained as $\mathcal{I}(1)$, and the AoF, which quantifies the severity of the fading, can be obtained as, AoF $=\frac{\mathcal{I}(2)-\mathcal{I}^{2}(1)}{\mathcal{I}^{2}(1)}=\frac{\mathcal{I}(2)}{\mathcal{I}^{2}(1)}-1$. It is also worth mentioning that the expression for the general moments of endto-end SINR is also very useful in the analysis of ergodic capacity of the system.

\subsection{Symbol error rate}

The average SER is another important performance measure. For a number of modulation schemes, the average SER admits the following expression, SER $=\int_{0}^{\infty} a_{\bmod } Q\left(\sqrt{2 b_{\bmod }}\right) f(\gamma) d \gamma$, where $Q(\cdot)$ is the Gaussian-Q function, $a_{\bmod }$ and $b_{\text {mod }}$ are modulation specific constants. For instance, binary phase shift keying $(\mathrm{BPSK})\left(a_{\bmod }=1, b_{\text {mod }}=1\right)$, binary frequency shift keying (BFSK) with orthogonal signalling $\left(a_{\bmod }=1\right.$, $\left.b_{\text {mod }}=0.5\right)$ and $M$-ary pulse amplitude modulation $(\mathrm{PAM})\left(a_{\mathrm{mod}}=2(M-1) / M, b_{\mathrm{mod}}=3 /\left(M^{2}-1\right)\right)$.

To evaluate the average SER, we find it convenient to use the following alternative expression [24]

$$
\operatorname{SER}=\frac{a_{\mathrm{mod}} \sqrt{b_{\mathrm{mod}}}}{2 \sqrt{\pi}} \int_{0}^{\infty} \frac{e^{-b_{\mathrm{mod}} \gamma}}{\gamma^{1 / 2}} \mathcal{F}_{\gamma_{\mathrm{d}}}(\gamma) d \gamma .
$$

Upon substituting Equation 6 into Equation (15), with the help of [[23], Eq. 7.621.6], we obtain the SER of interference-limited dual-hop AF relaying system in Nakagami- $m$ fading channels as

$$
\begin{aligned}
\text { SER } & =\frac{a_{\text {mod }} \sqrt{b_{\text {mod }}}}{2 \sqrt{\pi}}\left\{\sqrt{\frac{\pi}{b_{\text {mod }}}}-\left(\frac{m_{2} \bar{\gamma}_{l_{k}}}{\Omega_{2} m_{I_{k}}}\right)^{m_{2}} \sum_{i=0}^{m_{1}-1} \frac{1}{i !}\left(\frac{m_{1}}{\Omega_{1} \rho_{1}}\right)^{i} \sum_{j=0}^{i}\left(\begin{array}{l}
i \\
j
\end{array}\right)\left(\frac{\rho_{1} \Omega_{1}+1}{\rho_{2} N_{1}}\right)^{j}\right. \\
& \left.\times \sum_{k=1}^{N} \sum_{t=1}^{m_{l_{k}}} \frac{\beta_{k}^{t-1} \Gamma\left(a_{1}\right)}{(t-1) ! \Gamma\left(m_{2}\right)}\left(\frac{m_{1}\left(\rho_{1} \Omega_{1}+1\right)}{\Omega_{1} \rho_{1} \rho_{2} N_{1}}\right)^{m_{2}-j}\left(\frac{\Omega_{1} \Omega_{2} m_{I_{k}} \rho_{1} \rho_{2} N_{1}}{m_{1} m_{2}\left(\rho_{1} \Omega_{1}+1\right) \bar{\gamma}_{I_{k}}}\right)^{\zeta} \Xi_{1}\right\},
\end{aligned}
$$

where $\Xi_{1}$ is given by

$$
\Xi_{1}=\left\{\begin{array}{l}
\frac{\Gamma(\zeta) \Gamma(i+1 / 2)}{\Gamma\left(a_{1}+i+1 / 2\right)} F_{1} F_{1}\left(\zeta, i+1 / 2 ; a_{1}+i+1 / 2 ; 1-B\right),|1-B|<1, \\
\frac{\Gamma(\zeta) \Gamma(i+1 / 2)}{\Gamma\left(a_{1}+i+1 / 2\right)}\left(\frac{\left(m_{1}+\rho_{1} \Omega_{1} b_{\bmod }\right) \Omega_{2} m_{1} \rho_{2} N_{1}}{m_{1} m_{2}\left(\rho_{1} \Omega_{1}+1\right) \bar{Y}_{I}}\right)_{2}^{\zeta} F_{1}\left(a_{1}, \zeta ; a_{1}+i+\frac{1}{2} ; 1-\frac{1}{B}\right), B>1 / 2,
\end{array}\right.
$$

with

$$
B=\frac{m_{1} m_{2}\left(\rho_{1} \Omega_{1}+1\right) \bar{\gamma}_{I_{k}}}{\left(m_{1}+\rho_{1} \Omega_{1} b_{\mathrm{mod}}\right) \Omega_{2} m_{I_{k}} \rho_{2} N_{1}}
$$

$\zeta=m_{2}+i+\frac{1}{2}-j$. For special case, where $m_{I_{i}}=m_{I}$ and $\bar{\gamma}_{I_{i}}=\bar{\gamma}_{I}$, for $i=1, \ldots, N$, the SER of the system simplifies to

$$
\begin{aligned}
\operatorname{SER} & =\frac{a_{\bmod } \sqrt{b_{\bmod }}}{2 \sqrt{\pi}}\left\{\sqrt{\frac{\pi}{b_{\bmod }}}-\left(\frac{m_{2} \overline{\gamma_{1}}}{\Omega_{2} m_{l}}\right)^{m_{2}} \sum_{i=0}^{m_{1}-1} \frac{1}{i !}\left(\frac{m_{1}}{\Omega_{1} \rho_{1}}\right)^{i} \sum_{k=0}^{i}\left(\begin{array}{l}
i \\
k
\end{array}\right)\left(\frac{\rho_{1} \Omega_{1}+1}{\rho_{2} N_{1}}\right)^{k} \frac{\Gamma\left(b_{1}\right) \Gamma\left(b_{2}\right)}{\Gamma\left(N m_{l}\right) \Gamma\left(m_{2}\right)}\right. \\
& \left.\times\left(\frac{m_{1}\left(\rho_{1} \Omega_{1}+1\right)}{\Omega_{1} \rho_{1} \rho_{2} N_{1}}\right)^{m_{2}-k}\left(\frac{\Omega_{1} \Omega_{2} m_{1} \rho_{1} \rho_{2} N_{1}}{m_{1} m_{2}\left(\rho_{1} \Omega_{1}+1\right) \bar{\gamma}_{10}}\right)^{\xi} \Xi_{2}\right\},
\end{aligned}
$$

where $\Xi_{2}$ is given by

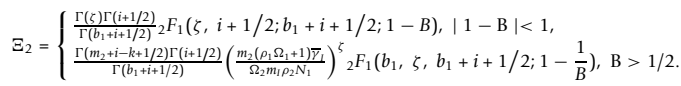

\subsection{Ergodic capacity}

Ergodic capacity, which is defined as the maximum error-free data rate that a channel can deliver, is another important performance metric. The ergodic capacity in (bits/seconds/hertz) is given by $C=\frac{1}{2} \mathrm{E}\left\{\log _{2}\left(1+\gamma_{d}\right)\right\}$, where $\frac{1}{2}$ accounts for the two phases required for the entire transmission. Due to the complicated form of the pdf of $\gamma_{d}$, the exact expression for the ergodic capacity is difficult to obtain. However, by noticing that $\log _{2}(x)$ is a concave function, tight capacity upper bound can be obtained by applying Jensen's inequality, i.e.,

$$
C \leq C_{\cup}=\frac{1}{2} \log _{2}\left(1+\mathrm{E}\left\{\gamma_{d}\right\}\right)=\frac{1}{2} \log _{2}(1+\mathcal{I}(1)) .
$$

Alternatively, accurate approximation of the ergodic capacity can be obtained by using the Taylor expansionbased method $[25,26]$, i.e.,

$$
C \approx C_{\mathrm{app}}=\frac{1}{2} \log _{2}(e)\left\{\ln \left(1+\mathrm{E}\left\{\gamma_{d}\right\}\right)+\frac{\left(\mathrm{E}\left\{\gamma_{d}^{2}\right\}-\mathrm{E}\left\{\gamma_{d}^{2}\right\}\right)}{2\left(1+\mathrm{E}\left\{\gamma_{d}\right\}\right)^{2}}\right\} .
$$

It is worth pointing out that the above approximation works quite well across the entire SNR range of interest.

We are also interested in how the received interference power distribution affects the ergodic capacity of the system, and we have the following key result.

Theorem 3: Let vector $r=\left[\bar{\gamma}_{I_{1}}, \ldots, \bar{\gamma}_{I_{N}}\right]$ denotes the average received interfering powers, when the interfering channels experience the same level of fading, i.e., 
$\left\{m_{I_{i}}\right\}_{i=1}^{N}=m_{I}$, then the ergodic capacity of the system is a Schur-convex function with respect to $r$. Thus, for any $r_{1}>r_{2}{ }^{\mathrm{c}}$

$$
C\left(r_{1}\right) \geq C\left(r_{2}\right) .
$$

Proof: See Appendix C.

Having established the schur-convexity for the ergodic capacity $C(\gamma)$, the following example illustrates how this can be used to address the impact of the received interference power distribution on the ergodic capacity performance for the relay systems in an interferencelimited environment.

Example 1: Let us consider the case with five co-channel interferers of a total received interference power of ten units in three different scenarios: (1) with only one effective interferer $\boldsymbol{r}_{1}=\left[\begin{array}{lllll}10 & 0 & 0 & 0 & 0\end{array}\right]$, (2) with distinctpower interferers $\boldsymbol{r}_{2}=\left[\begin{array}{lllll}6 & 2 & 1 & 0.75 & 0.25\end{array}\right]$, and (3) with equal-power interferers $\boldsymbol{r}_{3}=\left[\begin{array}{lllll}2 & 2 & 2 & 2 & 2\end{array}\right]$. Apparently, we have $\boldsymbol{r}_{1}>\boldsymbol{r}_{2}>\boldsymbol{r}_{3}$. Therefore, from Theorem 3 , we have

$$
C\left(r_{1}\right) \geq C\left(r_{2}\right) \geq C\left(r_{3}\right) .
$$

This example indicates that the best scenario occurs when there is only one interferer power at D, while the worst case happens when the interferers are of equal received power.

\section{Numerical results}

In this section, we provide numerical results to validate the analytical expressions derived from the previous section. Figure 1 illustrates the outage probability of the system when $\Omega_{1}=\Omega_{2}=2$, in the presence of three i.i.d. interferers with $m_{I}=3$ and $\gamma_{I}=5 \mathrm{~dB}$. The exact agreement between the analytical results and Monte Carlo simulation results indicates the correctness of the analytical expression. Also, we observe that the high SNR approximations work well even at moderate high SNR regime, and the diversity order of the system is determined by the minimum of $m_{1}$ and $m_{2}$.

In Figure 2, we fix the total channel power gain as $\Omega_{1}$ $+\Omega_{2}=4$, and investigate the impact of power imbalance between the two hops and fading effect of the interference links on the outage probability of the system. As we can observe, the outage probability of the system improves when the channel gain of the second hop increases, which is intuitive since the improvement of the second hop channel condition contributes more to suppressing the interference at the destination. Moreover, we see that the impact of fading level of the interference links on the outage performance depends on the SNR. For instance, for sufficiently high SNR, less severe fading interference links (i.e., large $m_{I}$ ) results in better outage performance, otherwise, more severe fading interference links (i.e., small $m_{I}$ ) becomes preferred in terms of outage probability.

Figure 3 shows the average SER performance of interference-limited dual-hop AF relaying systems employing BPSK modulation scheme with different number of interferers. For the simulation, we set $\Omega_{1}=2, m_{1}=3$, $\Omega_{2}=1.2, m_{2}=2, \bar{\gamma}_{I}=15 \mathrm{~dB}$ and $m_{I}=3$. It is easy to see that a single strong interferer significantly increases the SER of the system. In addition, when the number of

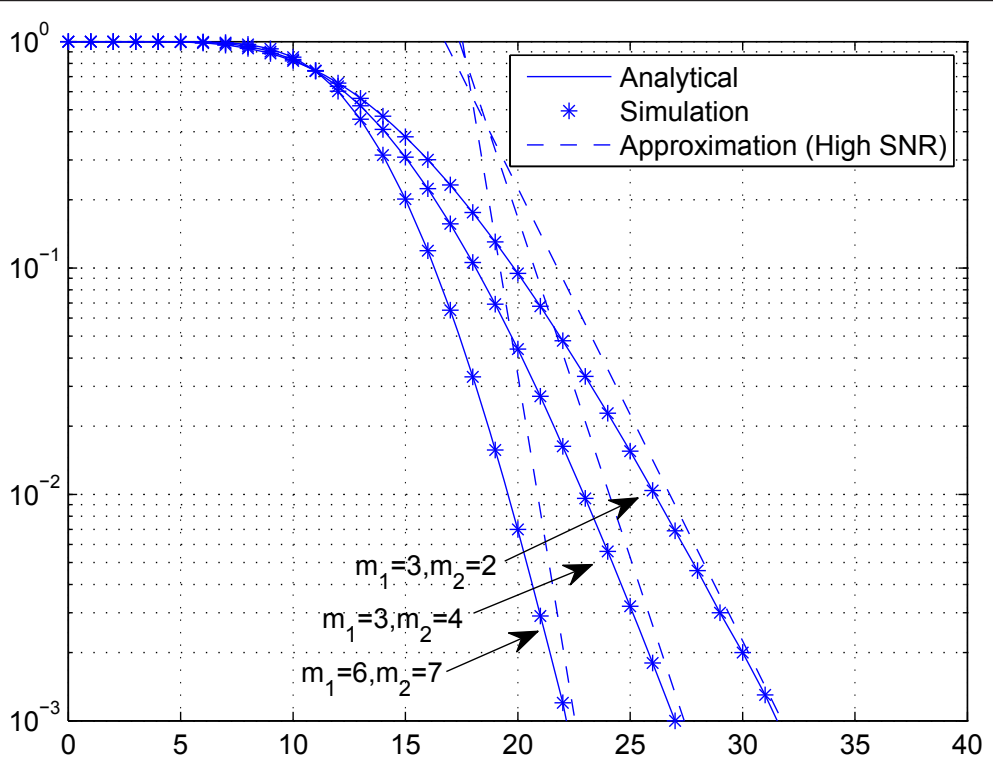

Figure 1 Outage probability of interference-limited dual-hop AF systems in Nakagami- $m$ fading channels when $\rho_{1}=\rho_{2}$ : exact versus high SNR approximation. 


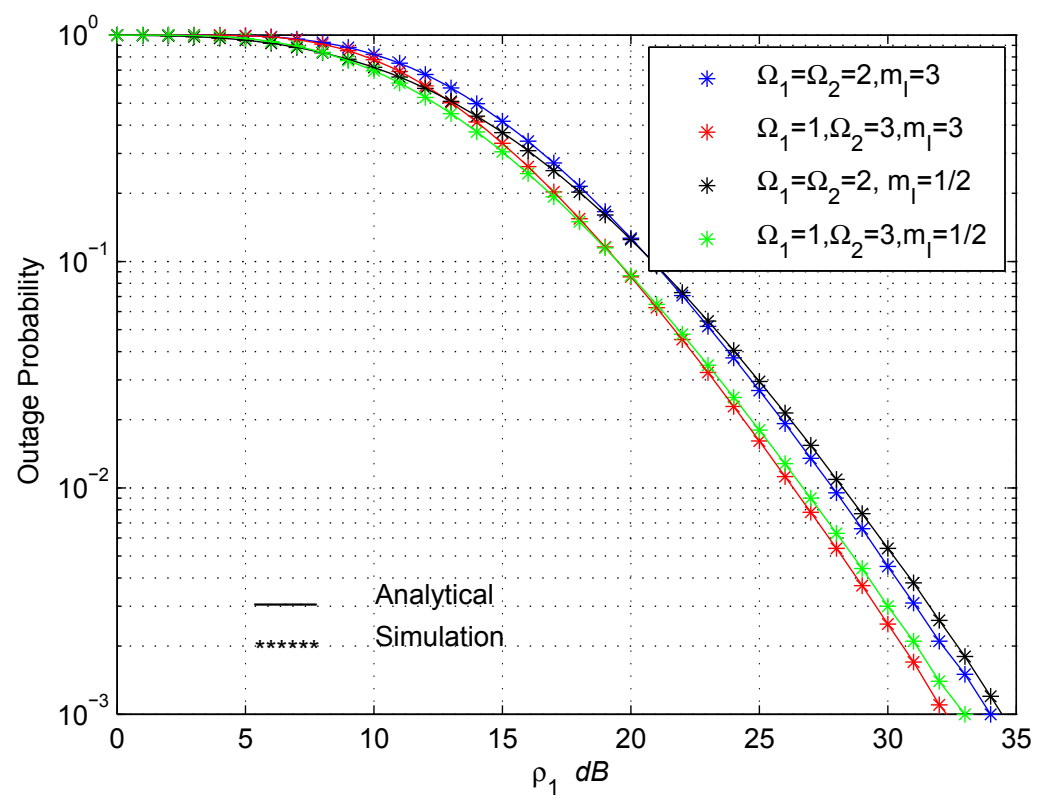

Figure 2 Outage probability of interference-limited dual-hop AF systems in Nakagami- $m$ fading channels with three i.i.d. interferers with $\gamma_{l}=5 \mathrm{~dB}$ when $\rho_{1}=\rho_{2}$.

interferers increases, hence the total interference power, the SER performance deteriorates further. Moreover, we see that when $\rho_{2}$ is fixed, the SER reaches an error floor, which is intuitive since the SER performance depends on both the first and second hop channels.

Figure 4 illustrates the ergodic capacity of the system. In this simulation, we assume four CCIs $N=4$ with average received power $\bar{\gamma}_{I}=8 \mathrm{~dB}$ fading parameter $m_{I}$ $=2$. The values of average channel gains and the fading parameters of the two hops are set to $\Omega_{1}=\Omega_{2}=2, m_{1}$ $=3$ and $m_{2}=2$. We observe that the capacity upper bound performs good at the low SNR regime. However, it gets loose gradually when the SNR increases. On the other hand, we see that the capacity approximation

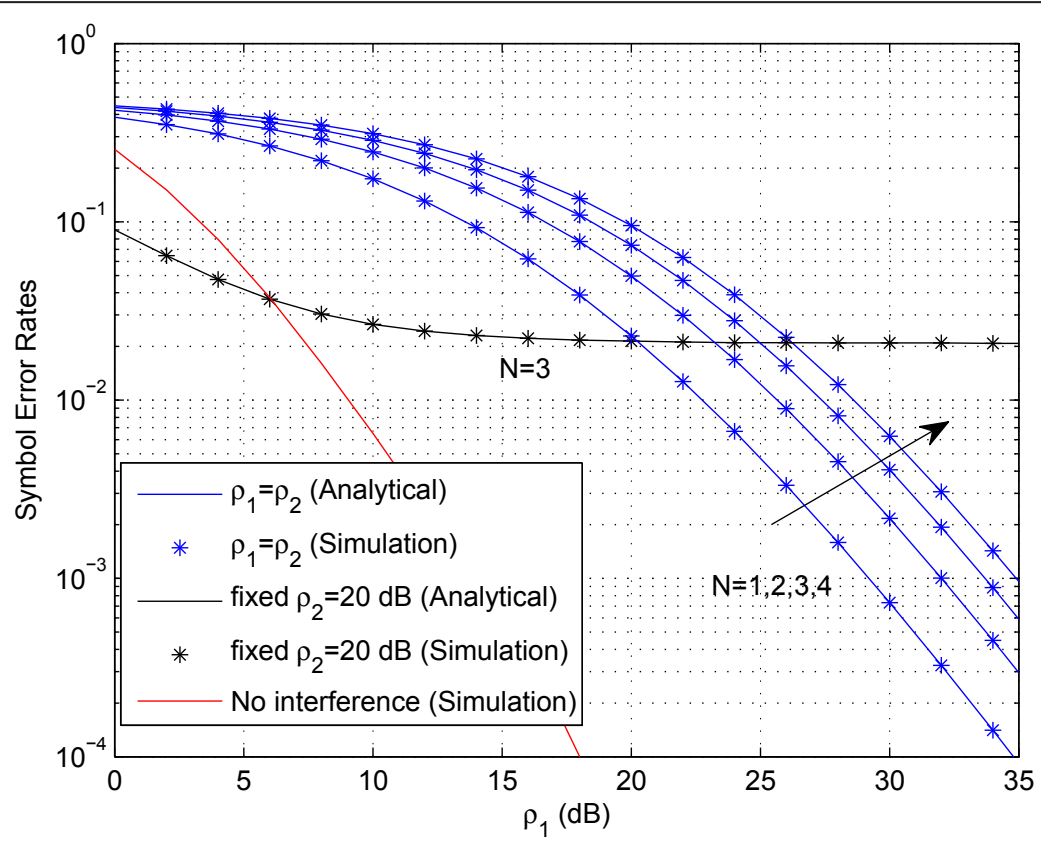

Figure 3 Symbol error probability performance of interference-limited dual-hop AF systems in Nakagami- $m$ fading channels. 


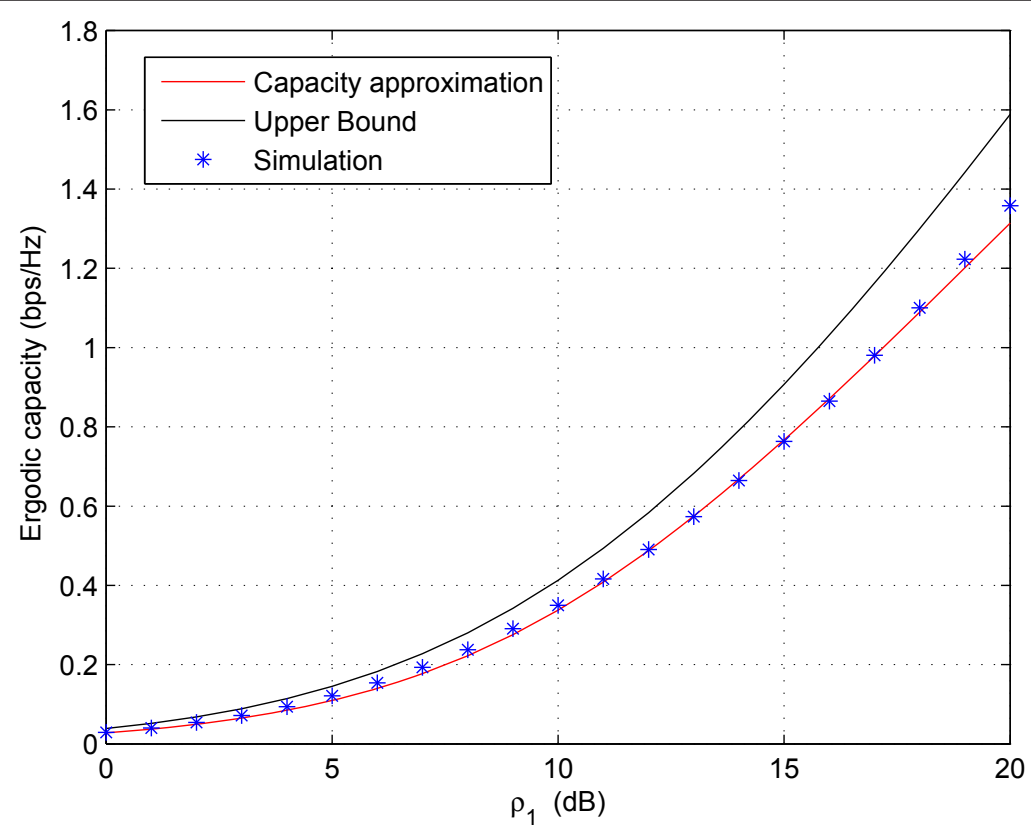

Figure 4 Ergodic capacity of interference-limited dual-hop AF relaying systems in Nakagami- $m$ fading channels when $\rho_{1}=\rho_{2}$ : capacity upper bound versus capacity approximation.

result is almost in perfect agreement with the exact result across the whole range of SNR regime of interest, hence it can be employed as an accurate and efficient means to evaluate the ergodic capacity of the system.

Figure 5 examines the impact of interference power distribution on the ergodic capacity of the system. In the simulation, we have assume that $\Omega_{1}=\Omega_{2}=1$, and
$m_{I}=1 / 2$. The curves provide intuitive result that the ergodic capacity of the system increases when the channel conditions of both hops improve, i.e., $m_{1}$ and $m_{2}$ become larger. Moreover, as predicted by Theorem 3, we observe that the highest capacity is achieved when there is only a single strong interferer, while the lowest

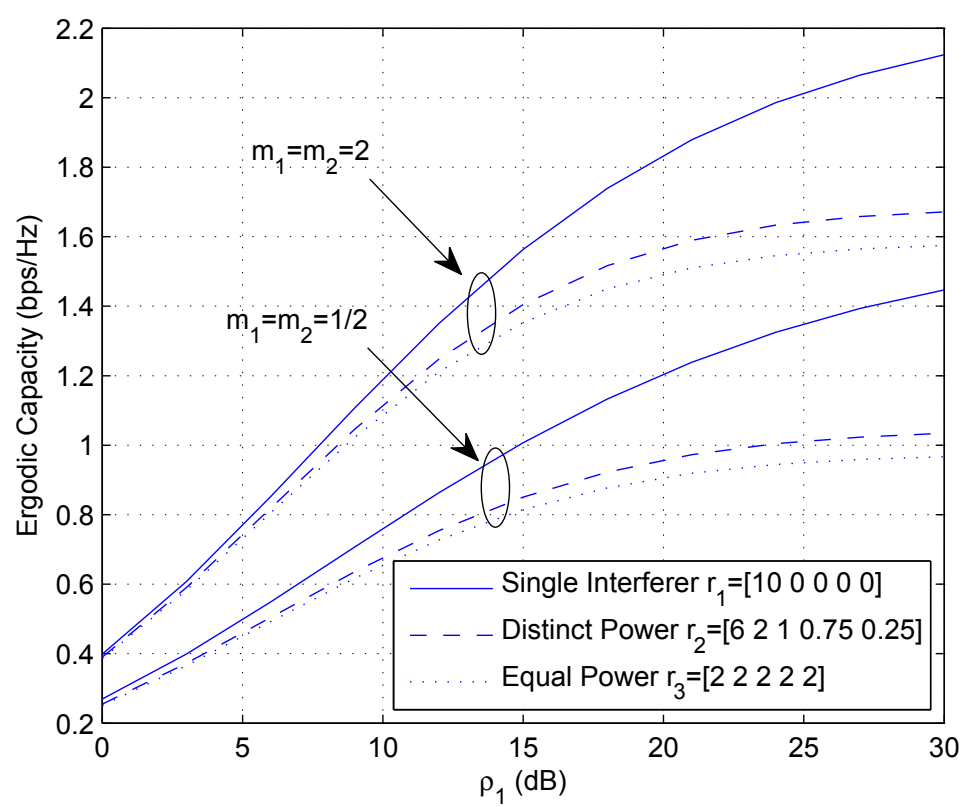

Figure 5 Impact of interference power distribution on the ergodic capacity of interference-limited dual-hop AF relaying systems in Nakagami- $m$ fading channels when $\rho_{2}=20 \mathrm{~dB}$. 
capacity is achieved when there are five equal power interferers.

\section{Conclusion}

In this article, we have provided a thorough investigation on the performance of interference-limited fixedagain AF dual-hop relaying systems in Nakagami- $m$ fading channels. Exact expressions were derived for the outage probability and average SER of the system as well as the general moments of the end-to-end SINR. Moreover, the diversity order and coding gain achieved the system were analyzed, and the results suggest that the diversity order of the system is determined by the channel experiencing severer fading and is not affected by the CCIs. In addition, we examined the ergodic capacity of the system, and showed that for a given total average received interference power at the destination, the scenario with a single strong interferer yields the best capacity performance.

\section{Appendix A}

\section{Proof of theorem 1}

In this section, we derive the c.d.f. expression of random variable $X=\frac{U_{1} U_{2}}{U_{2}+u Y}$. The p.d.f. and c.d.f. of Gamma random variables $U_{i}, \quad i=1,2$ are given by $f_{U_{i}}(\gamma)=\left(\frac{m_{i}}{\Omega_{i}}\right)^{m_{i}} \frac{\gamma^{m_{i}-1}}{\Gamma\left(m_{i}\right)} \exp \left(-\frac{m_{i}}{\Omega_{i}} \gamma\right), \quad$ and $\mathcal{F}_{U_{i}}(\gamma)=1-\exp \left(-\frac{m_{i}}{\Omega_{i}} \gamma\right) \sum_{j=0}^{m_{i}-1} \frac{1}{j !}\left(\frac{m_{i}}{\Omega_{i}} \gamma\right)^{j}, \quad \gamma>0$, respectively. $Y=\sum_{i=1}^{N} Y_{i}$, where $\left\{Y_{i}\right\}_{i=1}^{N}$ is a et of $N$ independent but not necessarily identically distributed (i.n.i.d.) gamma random variables with parameters $m_{I_{i}}$ and $\bar{\gamma}_{I_{i}}$. The p.d.f. of $Y$ is given in [13] as

$$
f_{Y}(\gamma)=\sum_{k=1}^{N} \sum_{i=1}^{m_{I_{k}}} \frac{\beta_{k}^{i-1} \gamma^{m_{I_{k}}}-1}{(i-1) !} \exp \left(-\frac{m_{I_{k}}}{\gamma_{I_{k}}} \alpha_{I_{k}} y\right) \text {, }
$$

where $\beta_{k}^{i-1}=\left.\frac{\prod_{i=1}^{N} \alpha_{I_{i}} m_{I_{i}}}{\left(m_{I_{k}}-i\right) !} \frac{d^{i-1}}{d s^{i-1}}\left[\prod_{\substack{n \neq k \\ n=1}}^{N}\left(\alpha_{I_{n}}+s\right)^{-m_{I_{n}}}\right]\right|_{s=\alpha_{k}}$. To this end, from the definition of c.d.f., we have $\mathcal{F}_{X}(x)=\operatorname{Pr}\left(U_{1}<\left(1+\frac{u Y}{U_{2}}\right) x\right)$. Conditioned on $U_{2}$ and $Y$, we first make use of the p.d.f expression of random variable $Y$ presented in (24), we have,

$$
\begin{aligned}
& \mathcal{F}_{X}\left(\left.x\right|_{U_{2}}\right)=1-\exp \left(-\frac{m_{1} x}{\Omega_{1}}\right) \sum_{i=0}^{m_{1}-1} \frac{1}{i !}\left(\frac{m_{1} x}{\Omega_{1}}\right)^{i} \sum_{j=0}^{i}\left(\begin{array}{l}
i \\
j
\end{array}\right)\left(\frac{u}{U_{2}}\right)^{j} \\
& \sum_{k=1}^{N} \sum_{t=1}^{m_{l_{k}}} \frac{\beta_{k}^{t-1}}{(t-1) !} \int_{0}^{\infty} Y^{m_{l_{k}}+k-1} \exp \left(-\left(\frac{m_{1} u x}{\Omega_{1} U_{2}}+\frac{m_{I_{k}}}{\bar{\gamma}_{I_{k}}}\right) Y\right) d Y,
\end{aligned}
$$

where the integral can be solved with the help of [[23], Eq.3.351.2]. Further averaging over $U_{2}$, we have the unconditional c.d.f. of $X$ as

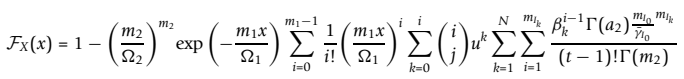

$$
\begin{aligned}
& \left(\frac{m_{1} u x}{\Omega_{1}}\right)^{-a_{2}} \int_{0}^{\infty}\left(1+\frac{\Omega_{1} m_{l_{0}}}{m_{1} u \bar{\gamma}_{0} x} U_{2}\right)^{-a_{2}} U_{2}^{m_{2}+m_{2}-1} \exp \left(-\frac{m_{2}}{\Omega_{2}} U_{2}\right) d U_{2} .
\end{aligned}
$$

The desired result can be obtained after some simple algebraic manipulations with the help of formula [[23], Eq. 3.383.5].

\section{Appendix B}

\section{Proof of theorem 2}

When $\rho_{1} \rightarrow \infty$, it is easy to see that $\frac{\rho_{1} \Omega_{1}+1}{\rho_{1}}=\Omega_{1}$, and $\exp \left(-\frac{m_{1} \gamma_{\text {th }}}{\Omega_{1} \rho_{1}}\right)=1$, therefore, the outage probability can be approximated as

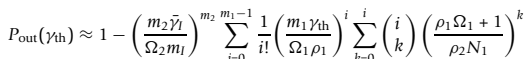

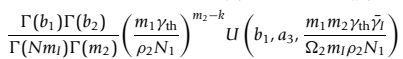

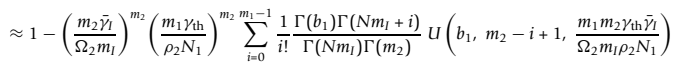

When $\rho_{2} \rightarrow \infty$, we are interested in the asymptotic behavior of $\rho_{2}^{-m_{2}} U\left(b_{1}, m_{2}-k+1, \frac{A}{\rho_{2}}\right)$. From the asymptotic expansion of function $U(a, b, x)$ given in [27], it is easy to observe that $\rho_{2}^{-m_{2}} U\left(b_{1}, m_{2}-k+1, \frac{A}{\rho_{2}}\right)$ approaches zero for $k \geq 1$. Therefore, the most significant term comes from $k=0$, which we compute as

$$
\rho_{2}^{-m_{2}} U\left(b_{1}, m_{2}+1, \frac{A}{\rho_{2}}\right) \approx \frac{\Gamma\left(m_{2}\right)}{\Gamma\left(b_{1}\right)} A^{-m_{2}} .
$$

To this end, the outage probability can be approximated as

$$
P_{\text {out }}\left(\gamma_{\text {th }}\right) \approx 1-\exp \left(-\frac{m_{1} \gamma_{\text {th }}}{\Omega_{1} \rho_{1}}\right) \sum_{i=0}^{m_{1}-1} \frac{1}{i !}\left(\frac{m_{1} \gamma_{\text {th }}}{\Omega_{1} \rho_{1}}\right)^{i} .
$$

Now we consider the case when $\rho_{1} \rightarrow \infty$ and $\rho_{2}=$ $\mu \rho_{1}$. We first focus on the scenario $m_{1}<m_{2}$. Conditioned on $\mathrm{X}_{2}$ and $Y$, the outage probability can be expressed as

$$
P_{\text {out }}\left(\gamma_{\text {th }} \mid X_{2}, Y\right)=\frac{\Gamma\left(m_{1}, \frac{m_{1} \gamma_{1,}}{\rho_{1} \Omega_{1}}\left(1+\frac{Y}{G^{2} N_{1} X_{2}}\right)\right)}{\Gamma\left(m_{1}\right)} \leq \frac{\left(\frac{m_{1} \gamma_{\text {th }}}{\rho_{1} \Omega_{1}}\left(1+\frac{Y}{G^{2} N_{1} X_{2}}\right)\right)^{m_{1}}}{\Gamma\left(m_{1}+1\right)} .
$$

A close observation reveals that the expectation of the right-hand side of Equation 30 converges as long as $m_{1}$ $<m_{2}$. Hence, we can exchange the order of limitation and integration, which gives

$$
\begin{aligned}
\lim _{\rho_{1 \rightarrow \infty} \rightarrow \infty} \mathrm{E}\left\{P_{\text {out }}\left(\gamma_{\text {th }} \mid X_{2}, Y\right)\right\} & =\mathrm{E}\left\{\lim _{\rho_{\mathrm{t}} \rightarrow \infty} P_{\text {out }}\left(\gamma_{\text {th }} \mid X_{2}, Y\right)\right\} \\
& =\frac{1}{\Gamma\left(m_{1}+1\right)}\left(\frac{m_{1}}{\Omega_{1}}\right)^{m_{1}}\left(\frac{\gamma_{\text {th }}}{\rho_{1}}\right)^{m_{1}} \mathrm{E}\left\{\left(1+\frac{\Omega_{1} Y}{\mu N_{1} X_{2}}\right)^{m_{1}}\right\},
\end{aligned}
$$


where we have used the following asymptotic expansion of the incomplete gamma function near zero

$$
\Gamma(a, x) \approx \frac{x^{a}}{a}, \quad x \rightarrow 0 .
$$

Due to the independence of $X_{2}$ and $Y$, we first apply the binomial expansion and compute the expectation in Equation 31 as follows,

$$
\mathrm{E}\left\{\left(1+\frac{\Omega_{1} Y}{\mu N_{1} X_{2}}\right)^{m_{1}}\right\}=\sum_{i=0}^{m_{1}}\left(\begin{array}{l}
m_{1} \\
i
\end{array}\right)\left(\frac{\Omega_{1}}{\mu N_{1}}\right)^{i} \mathrm{E}\left\{Y^{i}\right\} \mathrm{E}\left\{X_{2}^{-i}\right\} .
$$

To this end, the desired result can be obtained after some simple algebraic manipulations. The case for $m_{1}$ $>m_{2}$ can be dealt with in a similar fashion.

\section{Appendix C}

\section{Proof of theorem 3}

In order to prove Theorem 3, we need the following key lemma presented in [28].

Lemma 1: Let $G\left(p_{1}, \ldots, p_{n}\right) \triangleq \mathrm{E}\left[f\left(\sum_{k=1}^{n} p_{k} w_{k}\right)\right]$, where $f: \mathbb{R} \rightarrow \mathbb{R}$ with $f(x)>0 \forall x>0$, and $\left\{w_{i}\right\}_{i=1}^{n}$ are independent and identically distributed (i.i.d) positive random variables. If $f(x)$ is a convex function, then, $G(\boldsymbol{p})$ is a Schur-convex function with respect to $\boldsymbol{p} \triangleq\left(p_{1}, p_{2}\right.$, $\left.\ldots, p_{n}\right)$. On the other hand, if $f(x)$ is a concave function, then, $G(\boldsymbol{p})$ is Schur-concave with respect to $\boldsymbol{p}$.

According to the definition, we express the ergodic capacity of the system as

$$
C(\gamma)=\frac{1}{2} \mathrm{E}_{X_{1}, X_{2}, w_{i}}\left\{\log _{2}\left(1+\frac{\rho_{1} X_{1} X_{2}}{X_{2}+\frac{1}{\mathrm{G}^{2} N_{1}} \sum_{i=1}^{N} \bar{\gamma}_{I_{i}} w_{i}}\right)\right\},
$$

where $\left\{w_{i}\right\}_{i=1}^{N}$ are i.i.d. gamma random variables with parameter $m_{I}$ and 1 . To this end, we define function

$$
f(Z)=\log _{2}\left(1+\frac{\rho_{1} X_{1} X_{2}}{X_{2}+\mu Z}\right) .
$$

It is easy to show that the first and second derivatives of $f(Z)$ with respect to $Z$ can be expressed as

$$
f^{\prime}(Z)=-\frac{\rho_{1} X_{1} X_{2} \mu}{\left(X_{2}+\rho_{1} X_{1} X_{2}+\mu Z\right)\left(X_{2}+\mu Z\right)},
$$

and

$$
f^{\prime \prime}(Z)=\frac{\rho_{1} X_{1} X_{2} \mu^{2}\left(2 X_{2}+\rho_{1} X_{1} X_{2}+2 \mu Z\right)}{\left(X_{2}+\rho_{1} X_{1} X_{2}+\mu Z\right)^{2}\left(X_{2}+\mu Z\right)^{2}},
$$

respectively. Clearly, $f^{\prime}(Z) \geq 0$. According to Lemma 1, $C(\gamma)$ is Schur-convex function conditioned on $X_{1}$ and $X_{2}$, i.e., for any two vectors $\gamma_{1}>\gamma_{2}$, we have

$$
C\left(\gamma_{1} \mid X_{1}, X_{2}\right) \geq C\left(\gamma_{2} \mid X_{1}, X_{2}\right) .
$$

Averaging over $X_{1}$ and $X_{2}$, we have $C\left(\gamma_{1} \geq C\left(\gamma_{2}\right)\right.$. To this end, from the definition of Schur-convex function given in [29], we know that the ergodic capacity $C(\gamma)$ is an Schur-convex function with respect to $\gamma$.

\section{Endnotes}

${ }^{a}$ Here, we derived closedform expression for the sum of non-identical gamma random variables for the case where the fading parameters $m_{I_{i}}$ are distinct integers. For more generalized case, we can adopt the more general series representation, which applies for arbitrary $m_{I_{i}}[30]$. For such case, it is worth pointing out that the series converges quickly, and can be efficiently evaluated in standard software such as Mathematica. ${ }^{\mathrm{b}}$ The outage probability is originally defined as the probability that the system cannot support a given target rate $R_{\mathrm{th}}$. Hence, in this respect, the SINR threshold is related to the target rate by $\gamma_{t \mathrm{~h}}=2^{2 R_{\mathrm{th}}}-1 .^{\mathrm{c}}>$ denotes the majorization relationship. For details regarding majorization theory and Schur-convexity, we refer the readers to [29].

\section{Acknowledgment}

This work was supported by the Qatar National Research Fund (QNRF) grant through National Priority Research Program (NPRP) No. 08-577-2-241. QNRF

is an initiative of Qatar Foundation.

\section{Author details}

'Electrical \& Computer Engineering Program, Texas A\&M University at Qatar, Doha, Qatar ${ }^{2}$ The Institute of Information and Communication Engineering, Zhejiang University, Hangzhou, China ${ }^{3}$ The Institute of Electronics, Communications and Information Technology, Queen's University Belfast, Belfast, Northern Ireland

\section{Competing interests}

The authors declare that they have no competing interests.

Received: 1 May 2011 Accepted: 1 December 2011

Published: 1 December 2011

\section{References}

1. A Sendonaris, E Erkip, B Aazhang, User cooperation diversity-Part I: system description. IEEE Trans Commun. 51, 1927-1938 (2003). doi:10.1109/ TCOMM.2003.818096

2. JN Laneman, DNC Tse, GW Wornell, Cooperative diversity in wireless networks: efficient protocals and outage behavior. IEEE Trans Inf Theory. 50(12), 3062-080 (2004). doi:10.1109/TIT.2004.838089

3. MO Hasna, MS Alouini, End-to-end performance of transmission systems with relays over Rayleigh fading channels. IEEE Trans Wirel Commun. 2(6), 1126-131 (2003). doi:10.1109/TWC.2003.819030

4. MO Hasna, MS Alouini, A performance study of dual-hop transmissions with fixed gain relays. IEEE Trans Wirel Commun. 3(6), 1963-1968 (2004). doi:10.1109/TWC.2004.837470

5. TA Tsiftsis, GK Karagiannidis, SA Kotsopoulos, Dual-hop wireless communications with combined gain relays. IEE Trans Commun. 153(5), 528-532 (2005)

6. TA Tsiftsis, GK Karagiannidis, SA Kotsopoulos, F-N Pavlidou, BER analysis of collaborative dual-hop wireless transmissions. Electron Lett. 40(11), 679-681 (2004). doi:10.1049/el:20040393 
7. MO Hasna, MS Alouini, Harmonic mean and end-to-end performance of transmission systems with relays. IEEE Trans Commun. 52(1), 130-135 (2004). doi:10.1109/TCOMM.2003.822185

8. GK Karagiannidis, TA Tsiftsis, RK Mallik, Bounds for multihop relayed communications in Nakagami-m fading. IEEE Trans Commun. 54(1), 18-22 (2006)

9. S Ikki, MH Ahmed, Performance analysis of cooperative diversity wireless networks over Nakagami-m fading channel. IEEE Commun Lett. 11(4), 334-336 (2007)

10. DB Costa, MD Yacoub, Dual-hop transmissions with semi-blind relays over Nakagami-m fading channels. Electron Lett. 44(3), 214-216 (2008). doi:10.1049/el:20083507

11. GK Karagiannidis, Performance bounds of multihop wireless communications with blind relays over generalized fading channels. IEEE Trans Wirel Commun. 5(3), 498-503 (2006)

12. $\mathrm{S}$ Ikki, $\mathrm{MH}$ Ahmed, Performance analysis of dual-hop relaying communications over generalized Gamma fading channels, in Proc IEEE Globecom'07, Washington, DC, USA, pp. 3888-3893 (November 2007)

13. AA Abu-Dayya, NC Beaulieu, Outage probability of cellular mobile radio systems with multiple Nakagami interfers. IEEE Trans Veh Technol. 40(4), 757-768 (1991). doi:10.1109/25.108387

14. Q Zhang, Outage probability of cellular mobile radio in the presence of multiple Nakagami interferers with arbitrary fading parameters. IEEE Trans Veh Technol. 44(3), 661-67 (1995). doi:10.1109/25.406635

15. D Lee, I Lee, Outage probability for opportunistic relaying on multicell environment, in Proc IEEE VTC Spring 2009, Barcelona, Spain, pp. 1-5 (April 2009)

16. A Bletsas, AG Dimitriou, JN Sahalos, Interference-limited opportunistic relaying with reactive sensing. IEEE Trans Wirel Commun. 9(1), 14-20 (2010)

17. C Zhong, S Jin, KK Wong, Dual-hop system with noisy relay and interference-limited destination. IEEE Trans Commun. 58(3), 764-768 (2010)

18. HA Suraweera, HK Garg, A Nallanathan, Performance analysis of two hop amplify-and-forward systems with interference at the relay. IEEE Commun Lett. 14(8), 692-694 (2010)

19. AM Cvetković, GT Dordević, MC Stefanovć, Performance of interferencelimited dual-up non-regnerative relays over Rayleigh fading channels. IET Commun. 5(2), 135-140 (2011). doi:10.1049/iet-com.2010.0019

20. D Lee, JH Lee, Outage probability for dual-hop relaying systems with mutliple interferers over Rayleigh fading channels. IEEE Trans Veh Technol. 60(1), 333-338 (2011)

21. HA Suraweera, DS Michalopoulos, R Schober, GK Karagiannidis, N Arumugan, Fixed gain amplify-and-forward relaying with co-channel interference, in IEEE International Conference on Communications (ICC2011), (Kyoto, Japan)

22. F Al-Qahtani, T Duong, C Zhong, K Qaraqe, H Alnuweiri, Performance analysis of dual-hop AF systems with interference in Nakagami-m fading channels. IEEE Siganl Process Lett. 18(8), 454-457 (2011)

23. IS Gradshteyn, IM Ryzhik, Tables of Intergrals, Serious and Products, 6th edn. (Acadamic Press, San Diago, 2000)

24. MR McKay, A Grant, IB Collings, Performance analysis of MIMO-MRC in double-correlated Rayleigh environments. IEEE Trans Commun. 55(3), 497-507 (2007)

25. J Perez, I Ibanez, I Santamaria, Closed-form approximation for the outage capacity of orthogonal STBC. IEEE Commun Lett. 9(11), 961-963 (2005). doi:10.1109/LCOMM.2005.11001

26. DB Costa, S Aïssa, Capacity analysis of cooperative system with relay selection in Nakagami-m fading. IEEE Commun Lett. 13(9), 637-639 (2009)

27. M Abramowitz, IA Stegun, Handbooks of Mathematical Funcations with Formuals, Gmphs, and Mathemtical Tables, 10th edn. (US Department of Commerce-N. B. S., 1972)

28. H Boche, EA Jorswieck, On Schur-convexity of expectation of weighted sum of random variables with applications. J Inequal Pure Appl Math. 5(2), 1-14 (2004)

29. AW Marshall, I Olkin, Inequalities: Theory of Majorization and Its Applications (Academic Press, New York, 1979)

30. MS Alouini, A Abdi, M Kaveh, Sum of gamma variates and performance of wireless communication systems over Nakagami-fading channels. IEEE Trans Veh Technol. 50(6), 1471-1479 (2001). doi:10.1109/25.966578 doi:10.1186/1687-1499-2011-190

Cite this article as: Al-Qahtani et al.: Performance analysis of fixed-gain AF dual-hop relaying systems over Nakagami- $m$ fading channels in the presence of interference. EURASIP Journal on Wireless Communications and Networking 2011 2011:190.

\section{Submit your manuscript to a SpringerOpen ${ }^{\circ}$ journal and benefit from:}

- Convenient online submission

- Rigorous peer review

- Immediate publication on acceptance

- Open access: articles freely available online

- High visibility within the field

- Retaining the copyright to your article

Submit your next manuscript at $\gg$ springeropen.com 